\title{
Monte Carlo Simulation Techniques in a Decision Support System for Group Decision Making
}

\author{
ANTONIO JIMÉNEZ, ALFONSO MATEOS AND SIXTO RÍOS-INSUA \\ Department of Artificial Intelligence, Madrid Technical University, Campus de Montegancedo s/n, 28660 \\ Madrid,Spain (E-mails: ajimenez@fi.upm.es; amateos@fi.upm.es; srios@fi.upm.es)
}

\begin{abstract}
This paper describes a group decision support system based on an additive multi-attribute utility model for identifying a consensus strategy in group decision-making problems where several decision-makers or groups of decision-makers elicit their own preferences separately. On the one hand, the system provides procedures to quantify the DM's or group of DMs' preferences separately. This involves assessing the DM's or group of DMs' component utilities that represent their preferences regarding the respective possible attribute values and objective weights that represent the relative importance of the criteria. On the other hand, we propose Monte Carlo simulation techniques for identifying a consensus strategy. An iterative process will be carried out, where, after the simulations have been performed, the imprecise component utilities and weights corresponding to the different DMs or groups of DMs are tightened to output more meaningful information in the next simulations to achieve a consensus strategy. Finally, an application to the evaluation of remedial strategies for restoring contaminated aquatic ecosystems illustrates the usefulness and flexibility of this decision support tool.
\end{abstract}

\section{Introduction}

Multiple Criteria Decision Making (MCDM) techniques are promising tools for aiding groups to make decisions. Some examples of multi-criteria group decision support are described in Hwang and Lin (1987), Bui (1987) and Hämäläinen (1996). Iz and Gardiner (1993) give a survey of MCDM techniques and related Group Decision Support Systems (GDSS) that have been tested in cooperative decision-making situations.

In this paper we introduce a preliminary GDSS based on the Generic Multi-Attribute Analysis System $(G M A A)^{(1)}$, Jiménez (2002), Jiménez, Ríos Insua and Mateos (2003) and Mateos, Jiménez and Ríos Insua (2003). The above system is based on an additive multiattribute utility model and accounts for uncertainty about the strategy consequences and incomplete information concerning the quantification of the decision-makers' (DM) preferences, which leads to classes of utility functions for the different attributes and imprecise attribute weights for the decision. Consequently, the system is suitable for group decision-making, where individual conflicting views in a group of DMs can be captured through imprecise answers.

We assume that there are several DMs or groups of DMs, located at different places in our group decision-making problem. The objectives hierarchy and attributes associated with the 
lowest-level objectives of this hierarchy are established and the alternatives to be evaluated, as well as their consequences and possible uncertainty, have been identified. Each DM or group of DMs uses the GMAA system to elicit its own preferences. Thus, several weight intervals and classes of utility functions are available for the attributes under consideration.

We propose Monte Carlo simulation techniques for identifying a consensus strategy, in which the different inputs are randomly generated from the respective consequence, utility and weight intervals. The results of this technique can be easily analysed statistically by means of a multiple boxplot. An iterative process will be carried out, where, after the simulations have been performed, the imprecise component utilities and weights corresponding to the different DMs or groups of DMs are tightened to output more meaningful information in the next simulations to achieve a consensus strategy.

The paper includes three more sections. In Section 2, we describe the component utility and weight elicitation procedures provided by the GMAA system, which admits incomplete information. Section 3 deals with group decision-making where there are several DMs or groups of DMs with their own isolated preferences. An approach based on Monte Carlo simulation techniques will be introduced as a starting point for a negotiation process to achieve a consensus strategy. In Section 4, we present an application to the evaluation of remedial strategies for restoring contaminated aquatic ecosystems, where the preferences of two political parties were taken into account. Finally, in Section 5, some conclusions are provided.

\section{Quantification of DMs' Preferences}

The quantification of DM preferences involves assessing the component utilities that represent his/her preferences regarding the respective possible attribute values and objective weights that represent the relative importance of the criteria. Component utility functions can be used to identify efficient candidate solutions or to stimulate negotiation strategies, see, e.g., Jarke, Jelassi and Shakun (1987), Mumpower (1991), Verkama, Hämäläinen and Ethamo $(1992,1994)$ or Ethamo, Verkama and Hämäläinen (1994), as well as the attribute weights, see Hämäläinen (1996). Below, we introduce the methods provided by the GMAA (Generic Multi-Attribute Analysis) system, a DSS based on a multi-attribute utility model with imprecise assignment, to perform these tasks, see Jiménez (2002), Jiménez, Ríos Insua and Mateos (2003) or Mateos, Jiménez and Ríos Insua (2003).

The system admits incomplete information, allowing the DM to provide ranges of responses instead of precise values in the elicitation methods. This is less stressful on experts, since they are allowed to provide incomplete preference statements, see von Nitzsch and Weber (1988) and Ríos Insua (1994). Moreover, this makes the system suitable for group decision support, because individual conflicting views or judgements in a group of stakeholders can be captured through imprecise responses.

With respect to component utility assignment, the system provides four procedures: constructing a piecewise linear imprecise utility function, constructing an imprecise utility function with a gamble-based method, assigning imprecise utilities to discrete attribute values and directly providing subjective values. 


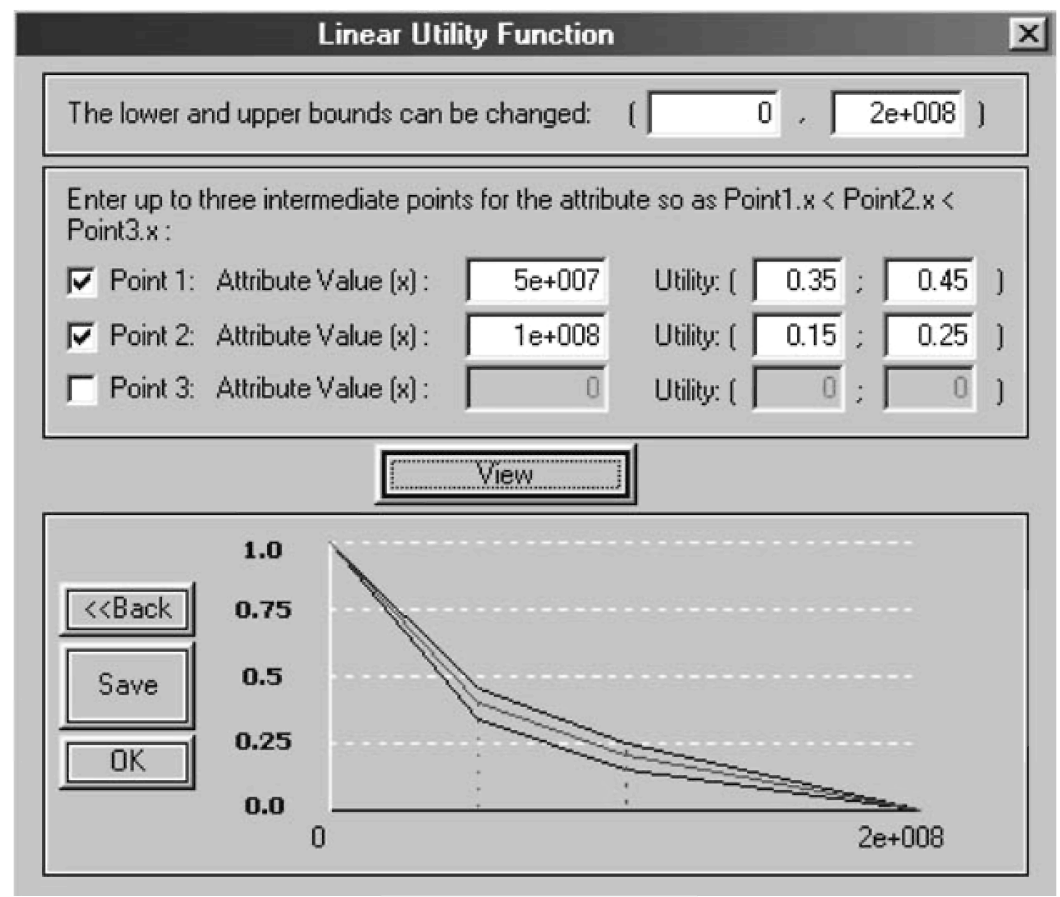

Figure 1. Constructing a piecewise linear imprecise utility function.

The first procedure available for assessing utility functions is to construct piecewise linear utility functions. The DM or DMs are asked to provide the best and the worst attribute values and up to three intermediate values with their respective imprecise utilities. The imprecise utility function will then be constructed by joining up to four linear segments between the best and the worst values. If no intermediate points are specified, then the result will be a single linear function. Figure 1 shows an example of the application of this procedure.

The second procedure available for constructing imprecise utility functions is based on the combination of two slightly modified standard methods: the fractile method and the extreme gambles method, which belong to the certainty equivalence (CE) and probability equivalence (PE) categories, respectively, see Farquhar (1984). As an innovation, both methods ask for imprecise rather than precise answers.

This assessment approach involves stating indifference conditions between gambles and sure amounts, and does not require the DM to indicate the form of the utility function.

Specifically, in the fractile method (Fishburn 1964, 1970; Hull, Moore and Thomas, 1973; Holloway 1979), the DM is asked to provide the certainty equivalence intervals or attribute value intervals $\left[x_{i}^{L}, x_{i}^{U}\right]$ that he/she considers equivalent to different gambles, whose results are the most and least preferred attribute values $x_{i}^{*}$ and $x_{i *}$ with probabilities $p$ and $1-p$, respectively. We take $p=0.25, p=0.50$ and $p=0.75$. This means that the DM considers 
the comparisons

$$
\left(\begin{array}{cc}
p & 1-p \\
x_{i}^{*} & x_{i *}
\end{array}\right) \sim x_{i}
$$

( $\sim$ means indifference) for all the amounts $x_{i} \in\left[x_{i}^{L}, x_{i}^{U}\right]$.

The method determines a range in the $x_{i} / u_{i}\left(x_{i}\right)$ diagram, which can be represented by the bounding value functions $u_{i}^{L_{\mathrm{CE}}}$ and $u_{i}^{U_{\mathrm{CE}}}$, where $L(U)$ means lower (upper).

In the extreme gambles method, (Mosteller and Nogee 1951; Schlaifer 1969), the DM has to specify probability intervals $\left[p^{L}, p^{U}\right]$ such that

$$
\left(\begin{array}{cc}
p & 1-p \\
x_{i}^{*} & x_{i *}
\end{array}\right) \sim x_{i}
$$

for all $p \in\left[p^{L}, p^{U}\right]$, for some selected amounts $x_{i}$ between $x_{i *}$ and $x_{i}^{*}$.

We use the upper bounds of the certainty equivalent intervals provided by the DM in the fractile method as sure amounts $x_{i}$. Other points may be used for comparison.

The system includes a routine implementing a wheel of fortune to output these probability intervals, see French (1986), which shows the probabilistic questions and guides the expert until an interval of indifference probabilities is obtained, see e.g. Figure 2. A number of additional questions are included as consistency checks.

If the intersection of the classes of utility functions output by the above methods were to be empty for some attribute values, the DM would have provided inconsistent responses and he/she should reassess his/her preferences. Thus, the intersection will be the range for the

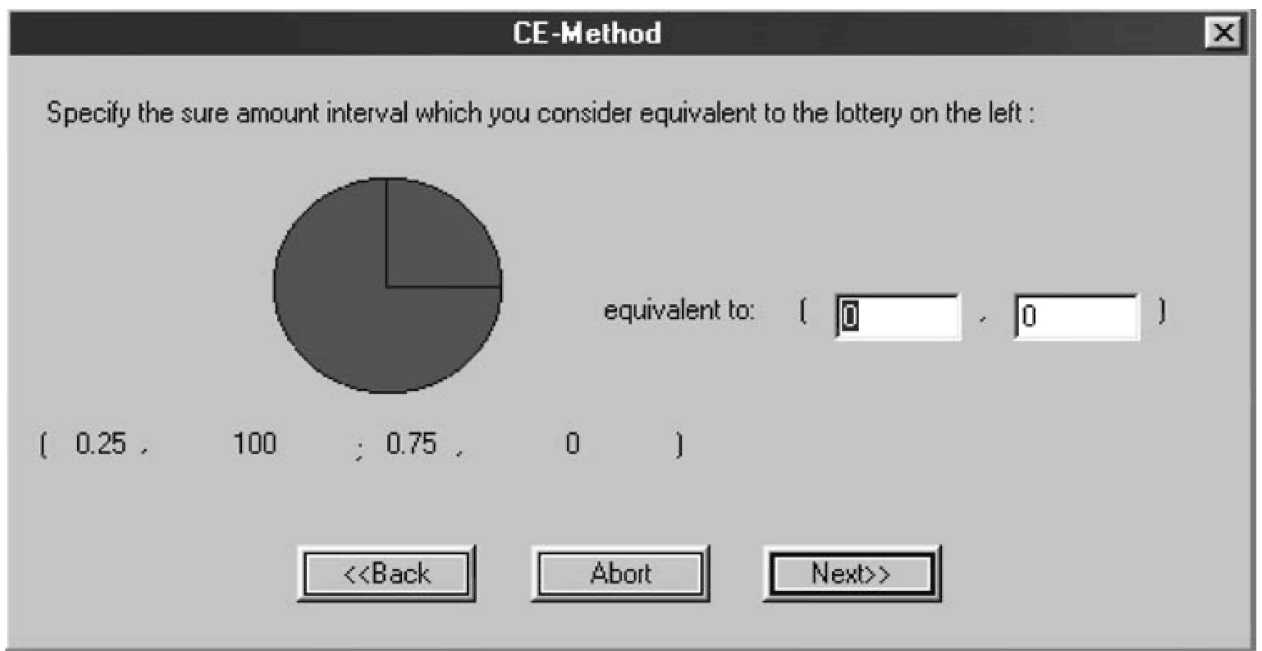

Figure 2. Constructing an imprecise utility function with the gamble-based method. 


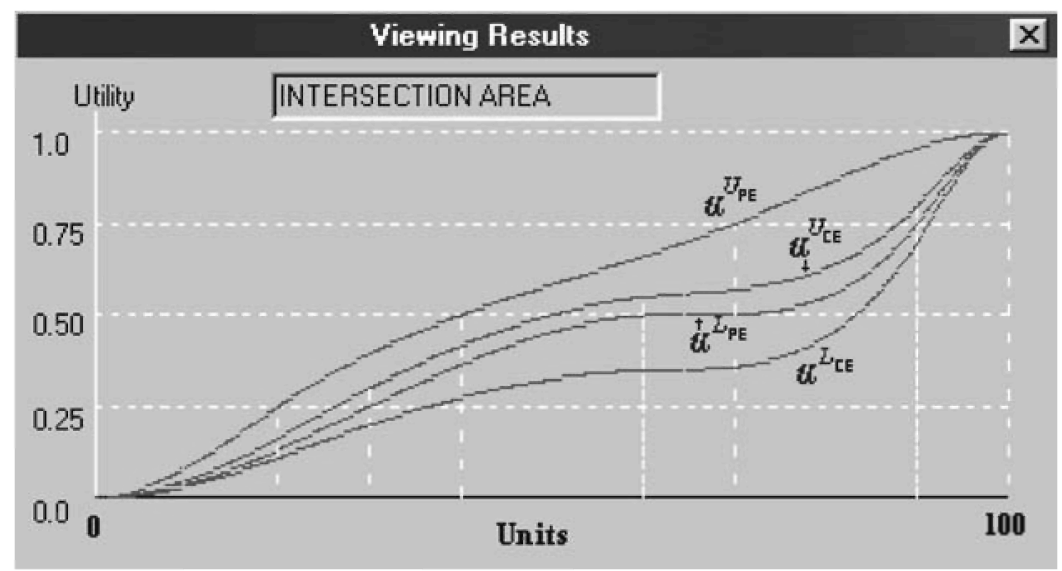

Figure 3. Intersection between classes of utility functions.

DM's utility functions, see Figure 3. The system is able to detect possible inconsistencies and suggests what the DM should change to achieve consistency.

The third procedure is used when different discrete attribute values have been identified for the attribute instead of a continuous scale. The DM or DMs are asked to provide the possible discrete attribute values (up to eight), their description and the respective imprecise utilities, see Figure 4.

Finally, the fourth procedure applies when the DM decides to use a subjective scale without defining a utility function and is implemented in the system by means of a thermometer scale, French et al. (1997). This procedure is suitable for determining the impact of each strategy on a given subjective attribute rather than a utility function, because it is difficult to ascertain what the value of each strategy is for the respective subjective attribute. For subjective scales, the DM will enter utility intervals by hand using scrollbars, as shown in Figure 5.

With respect to objective weight elicitation, the system provides two procedures: a method based on trade-offs and a direct assignment. The objective weights are elicited by weighting attributes and objectives along one branch at a time and multiplying the local weights through the objective hierarchy.

The first procedure is based on trade-offs among the respective attributes of the lowestlevel objectives stemming from the same objective, Keeney and Raiffa (1976). The DM is asked to give an interval of probabilities such that he/she is indifferent with respect to a lottery, whose results are the most and least preferred values of the attributes under consideration with the demanded probabilities $p_{i}$ and their complementary $1-p_{i}$, respectively, and certain sure consequences.

This procedure will be more suitable for the low-level objectives in the hierarchy because weight assessment involves a more specific area of knowledge. We begin with the objectives at the low levels of the hierarchy and then continue the assessment in ascending order of the hierarchy. 
Enter up to eight discrete attribute values so as the attribute value and upper utility for the first point is lower than for the second, and so on:

$\sqrt{\checkmark}$ Attribute Value 1: $\overline { 0 } \longdiv { \text { low level } }$ Utility: $(\overline{0.2}: 0.25: \sqrt{0.3}$ )

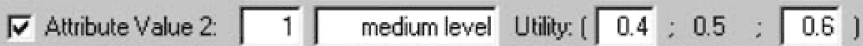

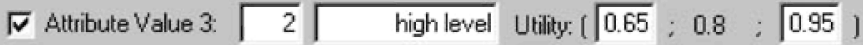

$\Gamma$ Attribute Value 4: $\overline { 0 } \longdiv { \quad \text { level4 } }$ Utility: $[\bar{\square} ; 0: \square$ ]

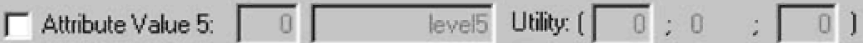

$\Gamma$ Attribute Value $6: \longdiv { 0 } \longdiv { \quad \text { lewel6 } }$ Utility: $(\sqrt{0}: 0: \sqrt{0}$ )

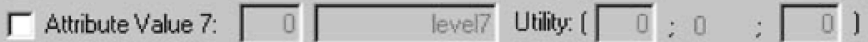

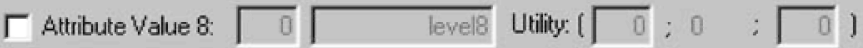

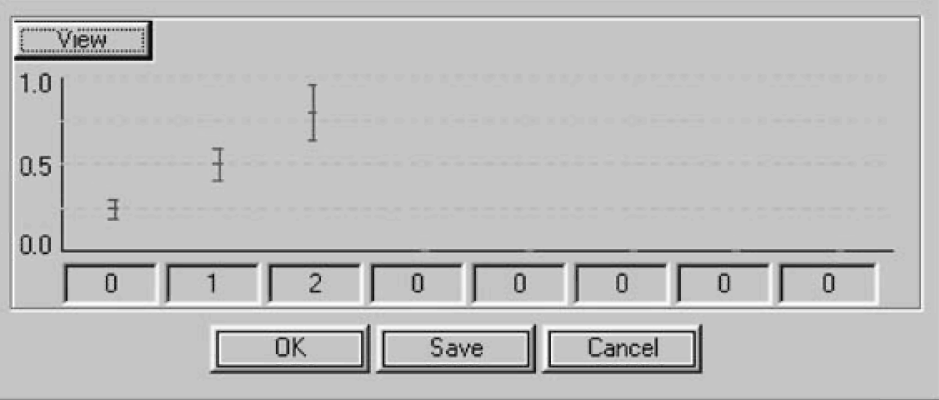

Figure 4. Imprecise utilities for discrete attribute values.

\section{Node Information}

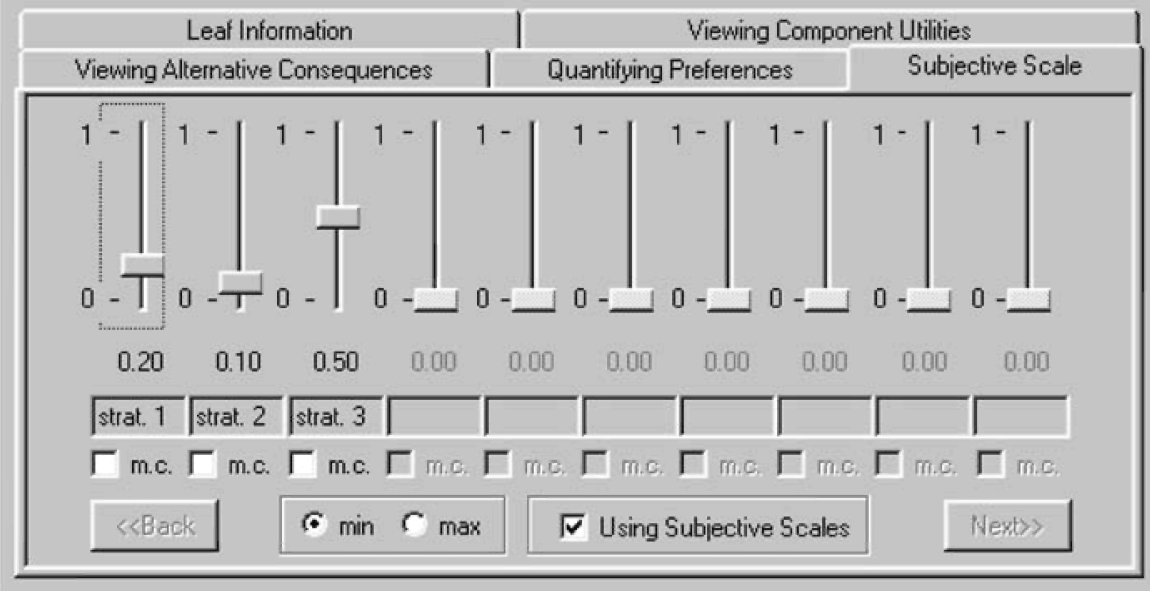

Figure 5. Subjective scale. 
The second procedure, perhaps more suitable for upper level objectives that could be more political, is based on direct assessment. Here, the DM is asked to directly provide weight intervals for the subobjectives under consideration, see Figure 6.

The system computes an average normalized weight and a normalized weight interval for each subobjective under consideration from the DM's responses through a normalization process. The user can use the radio buttons in the window shown in Figure 7 to see the respective average normalized weight and the endpoints of the normalized weight intervals.

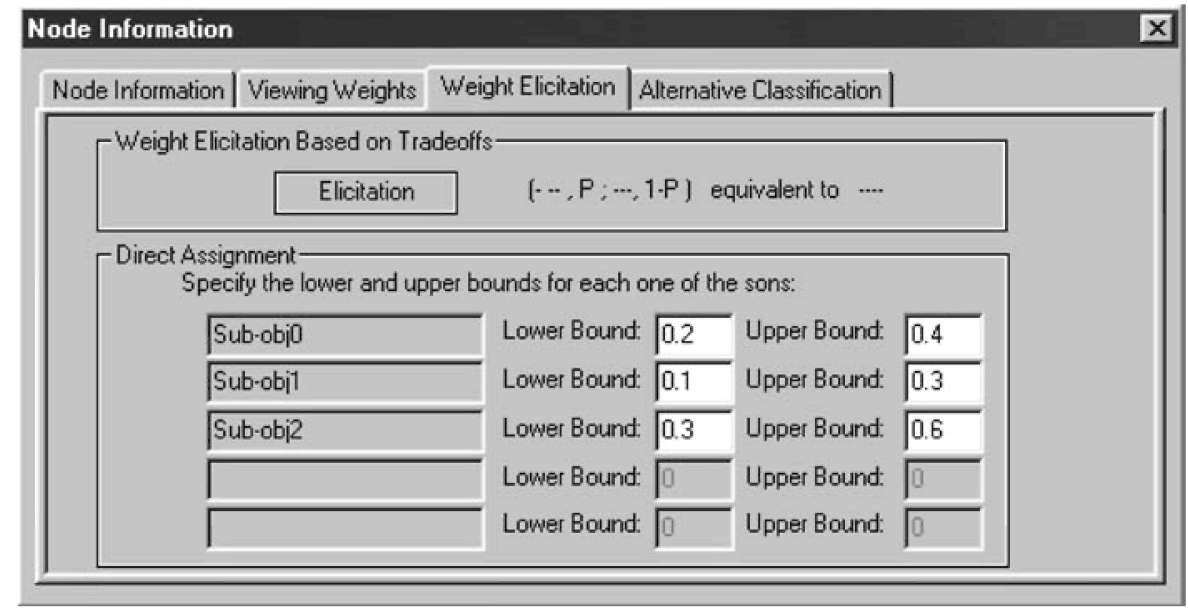

Figure 6. Direct assignment in weight elicitation.

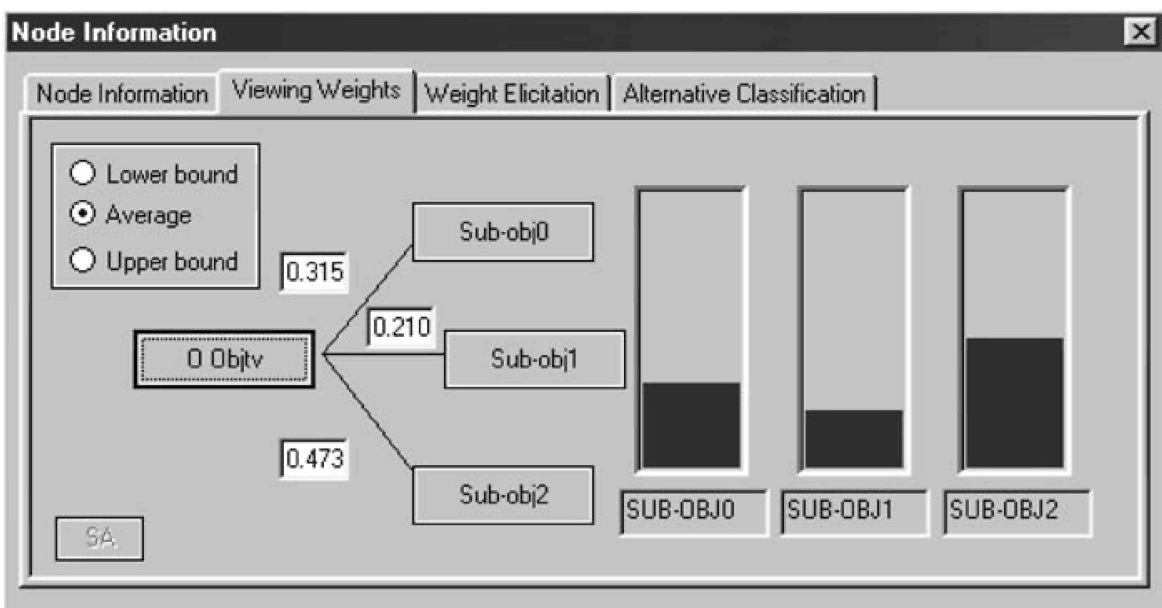

Figure 7. Average normalized weights and normalized weight intervals. 


\section{Monte Carlo Simulation Techniques for Group Decision-Making Support}

As mentioned above, we consider a group decision-making situation in which several DMs or groups of DMs elicit their own preferences separately. Hence, several classes of utility functions and weight intervals are available for each attribute under consideration, corresponding to the DM's or groups of DMs' preferences, obtained using the elicitation methods described in the above section.

We propose an approach based on Monte Carlo simulation techniques as a starting point for a negotiation process to achieve a consensus strategy.

We use a multiplicative linear congruential generator as a simulation program. This generator is based on Schrage's method, first published in 1979, and later refined in 1983, see Bratley, Fox and Schrage (1983). It provides a virtually infinite sequence of statistically independent random numbers, uniformly distributed between 0 and 1 .

Briefly, attribute weights are selected at random in each iteration, using the simulation program mentioned above, taking into account the DM's or groups of DMs' preferences, i.e., the respective weight intervals and their possible intersections. Next, the consequences for each strategy under consideration on the different attributes are selected at random. Then, their respective associated utilities are also selected at random, taking into account the classes of utility functions elicited from DMs or groups of DMs and their possible intersections.

Once we have the associated utilities for a specific strategy concerning the different attributes, the system computes and saves its overall utility on the basis of an additive multi-attribute utility model, taking the randomly generated attribute weights as well.

The system also computes the ranking of strategies and several statistics, like mode, minimum, maximum, mean, standard deviation and the 25 th, 50 th and 75 th percentiles, from their overall utilities. This information can be useful for discarding some possible strategies, aided by a display that presents a multiple boxplot for the strategy rankings.

The algorithm for the proposed simulation technique is as follows: Let $m$ be the number of simulations performed by the algorithm, $M$ the maximum number of simulations, $n$ the number of attributes in the decision-making problem and $s$ the number of strategies under consideration.

- For $m=1$ to $M$ :

- For $i=1$ to $n$ (for each attribute):

* Generate at random a weight for the $i$ th attribute, $k_{m i}^{R} \in I_{k_{i}}$, where $R$ means random and $I_{k_{i}}$ will be defined later.

- For $j=1$ to $s$ (for each alternative). 
* For $i=1$ to $n$ (for each attribute):

- Generate a random consequence from the imprecise consequence of strategy $S^{j}$ in the $i$-th attribute $x_{m i}^{j R} \in\left[x_{i}^{j L}, x_{i}^{j U}\right]$.

- From the random value $x_{m i}^{j R}$, generate a random utility, $u_{m i}^{j R}$, in the utility interval $I_{u}\left(x_{m i}^{j R}\right)$, where $I_{u}\left(x_{m i}^{j R}\right)$ will be defined later.

* Compute and save the overall utility of strategy $S^{j}: u_{m i}^{j R}=\sum_{i=1}^{n} k_{m i}^{R} u_{m i}^{j R}$.

- Compute the strategies ranking for the current simulation and update statistics.

- Display the resulting multiple boxplot.

There are two concepts to be clarified in the above algorithm, the determination of $I_{k_{i}}$ and $I_{u}\left(x_{m i}^{j R}\right) . I_{k_{i}}$ was defined when randomly generating a weight for the $i$-th attribute, $k_{m i}^{R}$, and represents its possible values taking into account the DM's or groups of DMs' preferences, i.e., their weight intervals. Next, we show how to determine $I_{k_{i}}$ when there are two and three DMs or groups of DMs. Then, in the general case with more than three DMs or groups of DMs, we will proceed similarly.

We could assume that there are no more or less important DMs or groups of DMs. On the other hand, a weight $w_{i}$ can be assigned to each DM or group of DMs representing its relative importance, $i=1, \ldots, d$, where $d$ is the number of DMs or groups of DMs and $\sum_{i=1}^{d} w_{i}=1$. Note that in the equally important case, $w_{1}=w_{2}=\cdots=w_{d}=\frac{1}{d}$.

If we consider two DMs or groups of DMs, whose elicited weights intervals for the $i$-th attribute are $\left[k_{i}^{1 L}, k_{i}^{1 U}\right]$ and $\left[k_{i}^{2 L}, k_{i}^{2 U}\right]$, respectively, then we will have the following two possibilities, see Figure 8:

1. If the intersection between the two weight intervals is empty, $k_{i}^{1 U}<k_{i}^{2 L}$, then $I_{k_{i}}=$ $\left[k_{i}^{1 L}, k_{i}^{1 U}\right] \cup\left[k_{i}^{2 L}, k_{i}^{2 U}\right]$ and $k_{m i}^{R}$ is randomly generated from $\left[k_{i}^{1 L}, k_{i}^{1 U}\right]$ with a probability of $w_{1}$ and from $\left[k_{i}^{2 L}, k_{i}^{2 U}\right]$ with a probability of $w_{2}$.

2. If the intersection between the two weight intervals is not empty at least at one point, i.e., $k_{i}^{1 L} \leq k_{i}^{2 L}<k_{i}^{1 U} \leq k_{i}^{2 U}$, then $I_{k_{i}}=\left[k_{i}^{2 L}, k_{i}^{1 U}\right]$ and $k_{m i}^{R}$ is randomly generated from the intersection interval.

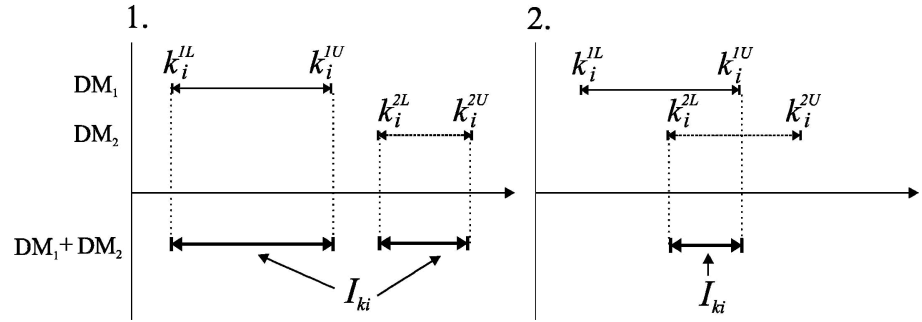

Figure 8. Determination of $I_{k_{i}}$ with two DMs or groups of DMs. 
1.

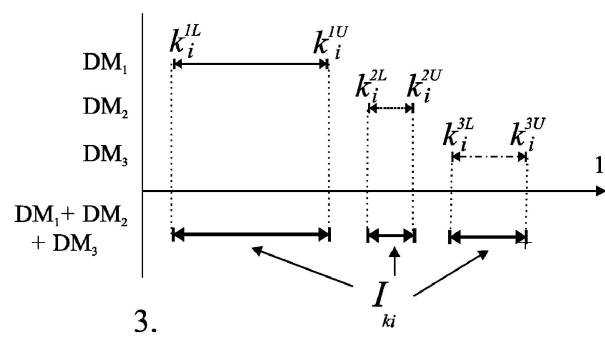

2.

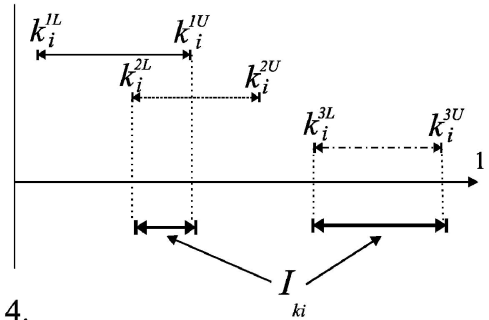

4.

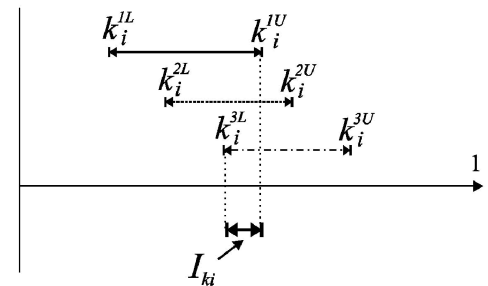

Figure 9. Determination of $I_{k_{i}}$ with three DMs or groups of DMs.

Another possibility would be to consider the union of both intervals. However, this is discarded, because, on the one hand, the intersection interval represents the weights on which all the DMs or groups of DMs agree and, on the other, as the interval width for the intersection is narrower than for the union, the intersection will lead to more meaningful or specific information in the multiple boxplot.

If we consider three DMs or groups of DMs, whose elicited weight intervals for the $i$-th attribute are $\left[k_{i}^{1 L}, k_{i}^{1 U}\right],\left[k_{i}^{2 L}, k_{i}^{2 U}\right]$ and $\left[k_{i}^{3 L}, k_{i}^{3 U}\right]$, respectively, we will now have the following four possibilities, see Figure 9:

1. If the intersection between any of the three weight intervals is empty, for instance, $k_{i}^{1 U}<k_{i}^{2 L}, k_{i}^{2 U}<k_{i}^{3 L}$, then $I_{k_{i}}=\left[k_{i}^{1 L}, k_{i}^{1 U}\right] \cup\left[k_{i}^{2 L}, k_{i}^{2 U}\right] \cup\left[k_{i}^{3 L}, k_{i}^{3 U}\right]$ and $k_{m i}^{R}$ is randomly generated with the probability of $w_{1}, w_{2}$ and $w_{3}$ from the weight intervals $\left[k_{i}^{1 L}, k_{i}^{1 U}\right],\left[k_{i}^{2 L}, k_{i}^{2 U}\right]$ and $\left[k_{i}^{3 L}, k_{i}^{3 U}\right]$, respectively.

2. If the intersection between two weight intervals is not empty and the third weight interval does no intersect with any of them, for instance, $k_{i}^{1 L} \leq k_{i}^{2 L}<k_{i}^{1 U} \leq k_{i}^{2 U}$ and $k_{i}^{2 U}<k_{i}^{3 L}$, then $I_{k_{i}}=\left[k_{i}^{2 L}, k_{i}^{1 U}\right] \cup\left[k_{i}^{3 L}, k_{i}^{3 U}\right]$ and $k_{i}^{R}$ is randomly generated with a probability of $w_{1}+w_{2}$ from the intersection weight interval, $\left[k_{i}^{2 L}, k_{i}^{1 U}\right]$, because this interval represents the weights on which these two DMs or groups of DMs agree, and with a probability of $w_{3}$ from the third weight interval, $\left[k_{i}^{3 L}, k_{i}^{3 U}\right]$, which represents the preferences of a single DM or group of DMs.

3. If a weight interval intersects with the other two but the intersection between the three is empty, for instance, $k_{i}^{1 L} \leq k_{i}^{2 L}<k_{i}^{1 U}<k_{i}^{3 L}<k_{i}^{2 U} \leq k_{i}^{3 U}$, then $I_{k_{i}}=\left[k_{i}^{2 L}, k_{i}^{1 U}\right] \cup\left[k_{i}^{3 L}, k_{i}^{2 U}\right]$ and $k_{m i}^{R}$ is randomly generated with the probability of 
$w_{1}+\frac{\left(k_{i}^{1 U}-k_{i}^{2 L}\right)}{\left(k_{i}^{1 U}-k_{i}^{2 L}\right)+\left(k_{i}^{2 U}-k_{i}^{3 L}\right)} w_{2}$ from the intersection weight interval, $\left[k_{i}^{2 L}, k_{i}^{1 U}\right]$ and with the probability of $w_{3}+\frac{\left(k_{i}^{2 U}-k_{i}^{3 L}\right)}{\left(k_{i}^{1 U}-k_{i}^{2 L}\right)+\left(k_{i}^{2 U}-k_{i}^{3 L}\right)} w_{2}$ from the intersection weight interval $\left[k_{i}^{3 L}, k_{i}^{2 U}\right]$.

4. If the three weight intervals intersect at least at one point, then $I_{k_{i}}=\left[k_{i}^{1 L}, k_{i}^{1 U}\right] \cap$ $\left[k_{i}^{2 L}, k_{i}^{2 U}\right] \cap\left[k_{i}^{3 L}, k_{i}^{3 U}\right]$ and $k_{m i}^{R}$ is randomly generated from the intersection weight interval.

For the general case with more than three DMs or groups of DMs, we will proceed similarly, and $k_{m i}^{R}$ will be randomly generated either from intersection intervals with probabilities depending on the number of intersected weight intervals or from single DM weight intervals. This leads us to a complex combinatorial problem that has been studied by different authors, with which, however, we will not deal in this paper.

With respect to $I_{u}\left(x_{m i}^{j R}\right)$, this set was defined when randomly generating a utility associated with a random strategy consequence $x_{i}^{j R}$ and represents its possible values taking into account the DM's or groups of DMs' preferences, i.e., their classes of utility function bounds at this point. Hence, given a random strategy consequence $x_{i}^{j R}, I_{u}\left(x_{m i}^{j R}\right)$ is determined similarly to $I_{k_{i}}$, but, in this case, we have utility rather than weight intervals. For instance, Figure 10 shows this analogy for a case with two DMs or groups of DMs.

The example in Figure 10 corresponds with the case where the intersection between the two utility intervals is empty and $u_{m i}^{j R}$ is randomly generated from $\left[u_{i}^{1 L}, u_{i}^{1 U}\right]$ with a

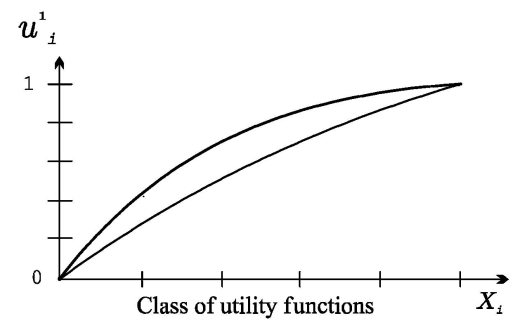

for DM 1

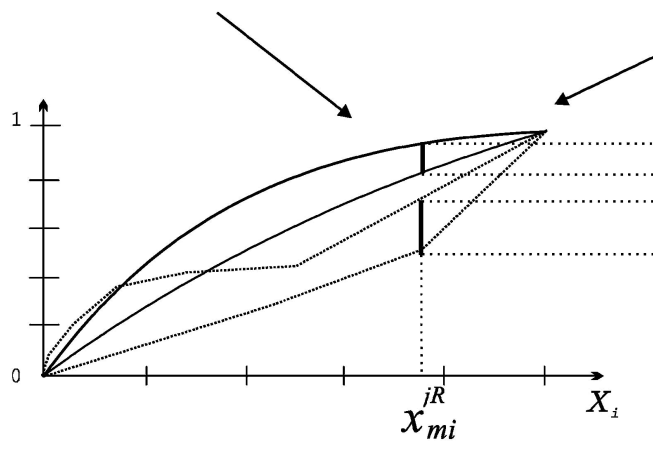

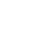

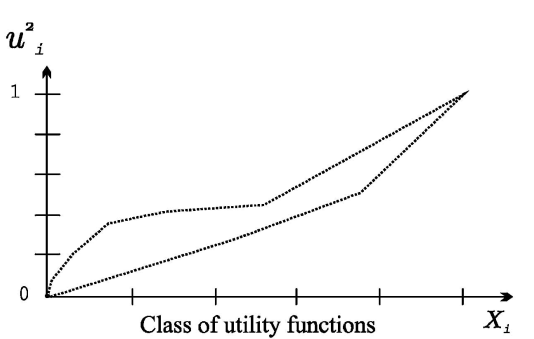

for DM 2

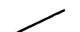

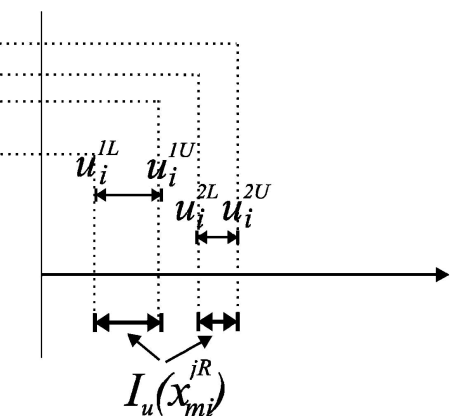

Figure 10. Determination of $I_{u}\left(x_{m i}^{j R}\right)$ with two DMs or groups of DMs. 


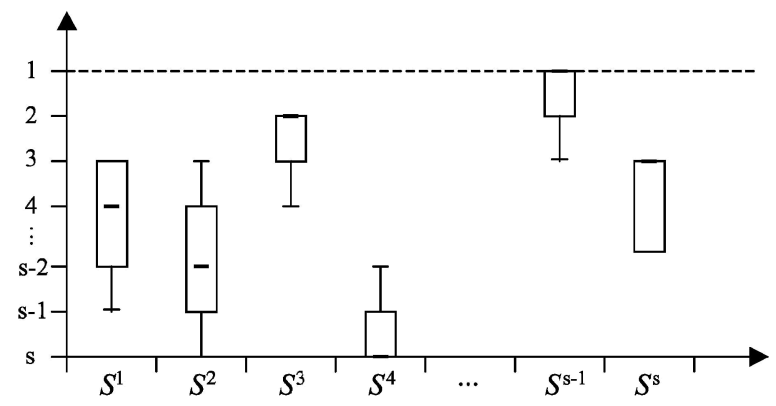

Figure 11. Resulting multiple boxplot.

probability of $w_{1}$ and from $\left[u_{i}^{2 L}, u_{i}^{2 U}\right]$ with a probability of $w_{2}$, assuming that the DMs or groups of DMs are equally important.

If there is an attribute with several discrete values, then the strategy consequences are assumed to be precise. In this case, $u_{m i}^{j R}$ is randomly generated in the same way as when there are imprecise utility functions, taking into account the possible intersection between the utility intervals corresponding to the respective discrete attribute values, as in the case of a subjective scale, where the subjective intervals are analyzed.

Finally, as mentioned above, when the simulation is run, the system computes several statistics about the rankings of the strategies in question, like mode, minimum, maximum, mean, standard deviation and the 25th, 50th and 75th percentiles. This information is displayed as a multiple boxplot for the strategies, see Figure 11. This can be useful for identifying the most preferred or "consensus" strategy. However, in most cases, the information inferred by the multiple boxplot is not meaningful enough to definitively recommend a strategy due to imprecision concerning the group decision-making problem, which leads to a negotiation process.

It is clear that the less imprecise the respective sets $I_{k_{i}}$ and $I_{u}\left(x_{m i}^{j R}\right)$ are in the simulation process, the more meaningful the information displayed in the multiple boxplot will be. Thus, the negotiation process will focus on reducing imprecision concerning $I_{k_{i}}$ and $I_{u}\left(x_{m i}^{j R}\right)$. This can be done if the DMs or groups of DMs are able to change their preferences to reach a consensus, i.e., the intersection between their respective weight intervals, classes of utility functions or both become non empty or a large non-empty intersection is tightened.

In this respect, we propose an attribute divergence index that guides the negotiation process after the simulation process has been performed. This index reports about the attributes whose preferences should be revised first to the DMs. The divergence index for the $i$-th attribute, $\mathrm{ADI}_{i}$, is defined as

$$
\mathrm{ADI}_{i}=\max _{j=1, \ldots, s}\left\{u_{i}^{j \max }-u_{i}^{j \min }\right\}
$$

where $u_{i}^{j \max }=\max _{m}\left\{k_{m i}^{R} u_{m i}^{j R}\right\}$ and $u_{i}^{j \min }=\min _{m}\left\{k_{m i}^{R} u_{m i}^{j R}\right\}$. 
Thus, the attributes will be revised in decreasing order with respect to the divergence index, i.e., the attribute with the highest divergence index is the attribute to be revised first and the attribute with the lowest divergence index is the attribute to be revised last.

If we include the assessment of the attribute divergence indexes in the algorithm for the proposed simulation technique, its appearance is as follows:

- Initialization:

- For $i=1$ to $n$ (for each attribute):

$* \mathrm{ADI}_{i}=0$

* For $j=1$ to $s$ (for each alternative):

- $u_{i}^{j \max }=0$.

- $u_{i}^{j \min }=1$.

- For $m=1$ to $M$ :

- For $i=1$ to $n$ (for each attribute):

* Generate at random a weight for the $i$-th attribute, $k_{m i}^{R} \in I_{k_{i}}$, where $R$ means random and $I_{k_{i}}$ is as defined previously.

- For $j=1$ to $s$ (for each alternative):

* For $i=1$ to $n$ (for each attribute):

- Generate a random consequence from the imprecise consequence of strategy $S^{j}$ in the $i$-th attribute $x_{m i}^{j R} \in\left[x_{i}^{j L}, x_{i}^{j U}\right]$.

- From the random value $x_{m i}^{j R}$, generate a random utility, $u_{m i}^{j R}$, in the utility interval $I_{u}\left(x_{m i}^{j R}\right)$, where $I_{u}\left(x_{m i}^{j R}\right)$ is as defined previously.

- Update values:

$$
\text { - If }\left(k_{m i}^{R} u_{m i}^{j R}>u_{i}^{j \max }\right) \text {, then } u_{i}^{j \max }=k_{m i}^{R} u_{m i}^{j R} .
$$$$
\text { - If }\left(k_{m i}^{R} u_{m i}^{j R}<u_{i}^{j \min }\right) \text {, then } u_{i}^{j \min }=k_{m i}^{R} u_{m i}^{j R} \text {. }
$$

- Compute and save the overall utility of strategy $S^{j}: u_{m}^{j R}=\sum_{i=1}^{n} k_{m i}^{R} u_{m i}^{j R}$.

* If $\left(\mathrm{ADI}_{i}<u_{i}^{j \max }-u_{i}^{j \min }\right)$, then $\mathrm{ADI}_{i}=u_{i}^{j \max }-u_{i}^{j \min }$.

- Compute the strategies ranking for the current simulation and update statistics.

- Display the resulting multiple boxplot.

Therefore, through a process of iteration and based on the divergence indexes, the DMs or groups of DMs can revise their preferences and the simulation process can be performed 
until the information displayed by the multiple boxplot is meaningful enough to identify a consensus strategy.

\section{An Illustrative Example}

As an example of the application of the approach based on Monte Carlo simulation techniques, introduced in the above section, we consider a complex political group decisionmaking problem, in which several political parties are involved. They must choose which strategy should be applied for the restoration of an aquatic ecosystem contaminated by radionuclides, Lake Kozhanovskoe, in the region of Bryansk (Russia), which was heavily contaminated with ${ }^{137} \mathrm{Cs}$ after the Chernobyl accident in 1986.

This complex decision-making problem was studied in depth in the 1998-2001 European Commission project of the international cooperation program (INCO-COPERNICUS) COMETES: Implementing Computerised METhodologies to Evaluate the effectiveness of countermeasures for restoring radionuclide contaminated fresh water ecoSystems, in which we participated, see, e.g., Gallego et al. (2001).

An objective hierarchy was built for this decision-making problem, which intended to provide the grounds for the description and evaluation of the hypothetical restoration alternatives for the scenario in question, see Figure 12, and attributes were established for the eight lowest-level objectives:

1. Ecosystem Index $\left(X_{1}\right)$. The ecosystem index is a measure of the ecosystem health in aquatic environments. It summarizes the state of important ecosystem variables affecting phytoplankton, benthos and fish.

2. Dose to Fish $\left(X_{2}\right)$. An assessment of the absorbed radiation dose received by fish as a consequence of the radioactive contamination of the aquatic ecosystem.

3. Dose to Critical Individual $\left(X_{3}\right)$. The effective dose received by individuals belonging to a critical group living in the area, drinking water, and eating aquatic food and terrestrial food irrigated with water from the contaminated water body.

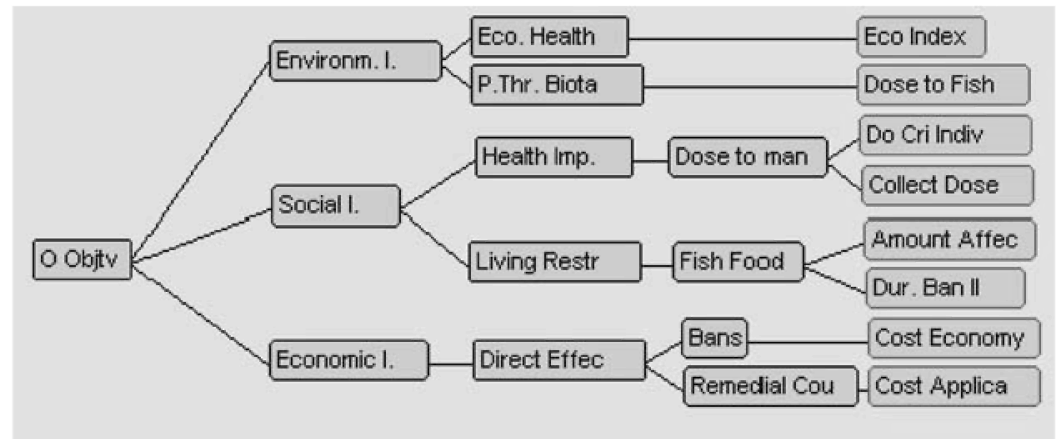

Figure 12. Objective hierarchy for the restoration problem. 
4. Collective Dose $\left(X_{4}\right)$. The effective collective dose received by all the population through all the exposure pathways due to the contaminated water body. It is a measure of the increased risk of serious latent health effects.

5. Amount Affected $\left(X_{5}\right)$. In the case of restrictions on fish consumption and food industry processing, the amount of fish affected by restrictions.

6. Duration of $B$ an $I I\left(X_{6}\right)$. In the case of restrictions on fish consumption and food industry processing, the duration of the restrictions.

7. Cost to Economy $\left(X_{7}\right)$. The direct economic impact of the restrictions, either in terms of the cost of the food affected by bans or in terms of the production lost (e.g., share of Gross Domestic Product Lost).

8. Cost of Application $\left(X_{8}\right)$. In the case of remedial countermeasures (physical or chemical), this represents the direct cost of the application: manpower, consumables, equipment needed for application, management of wastes generated, etc.

Table 1 shows these attributes as well as their measurement units and the respective ranges.

The restoration strategies to be analyzed were:

$S^{1}$ : No Action. Natural evolution of the situation without intervention.

$S^{2}$ : Potash Treatment. Reduction of aquatic organism uptake by potash treatment of aquatic ecosystems contaminated by radiocesium.

$S^{3}$ : Fertilization. Tonnes of fertilizer added to the lake to increase biomass (biological dilution).

$S^{4}$ : Lake Liming. Reduction of radionuclide remobilisation from sediments.

$S^{5}$ : Sediment Removal. $6 \mathrm{~km}^{2}$ of sediments removed from the lake down to a depth of $5 \mathrm{~cm}$.

$S^{6}$ : Automatic Food Bans. Automatic fish consumption ban when ${ }^{137} \mathrm{Cs}$ content in fish is greater than $1000 \mathrm{~Bq} / \mathrm{kg}$.

The two main local political parties were asked to participate in the decision-making problem, aided by decision analysts. Both parties were considered equally important, though

Table 1. Attributes used in evaluating the strategies.

\begin{tabular}{llll}
\hline & & \multicolumn{2}{c}{ Range } \\
\cline { 3 - 4 } Attribute & Unit of Measurement & Worst $x_{i *}$ & Best $x_{i}^{*}$ \\
\hline$X_{1}:$ Ecosystem Index & LEI (Ecosystem Index) & 5 & 1 \\
$X_{2}:$ Dose to Fish & mGy (MiliGray) & $10^{4}$ & 0 \\
$X_{3}:$ Dose to Critical Individual & mSv (miliSievert) & 500 & 0 \\
$X_{4}:$ Collective Dose & mSv $\times$ person & $12 \times 10^{4}$ & 0 \\
$X_{5}:$ Amount Affected & Tonnes & $10^{4}$ & 0 \\
$X_{6}:$ Duration of Ban II & Months & 120 & 0 \\
$X_{7}:$ Cost to Economy & Euros $\times 10^{2}$ & $10^{8}$ & 0 \\
$X_{8}:$ Cost of Application & Euros $\times 10^{2}$ & $2 \times 10^{8}$ & 0 \\
\hline
\end{tabular}




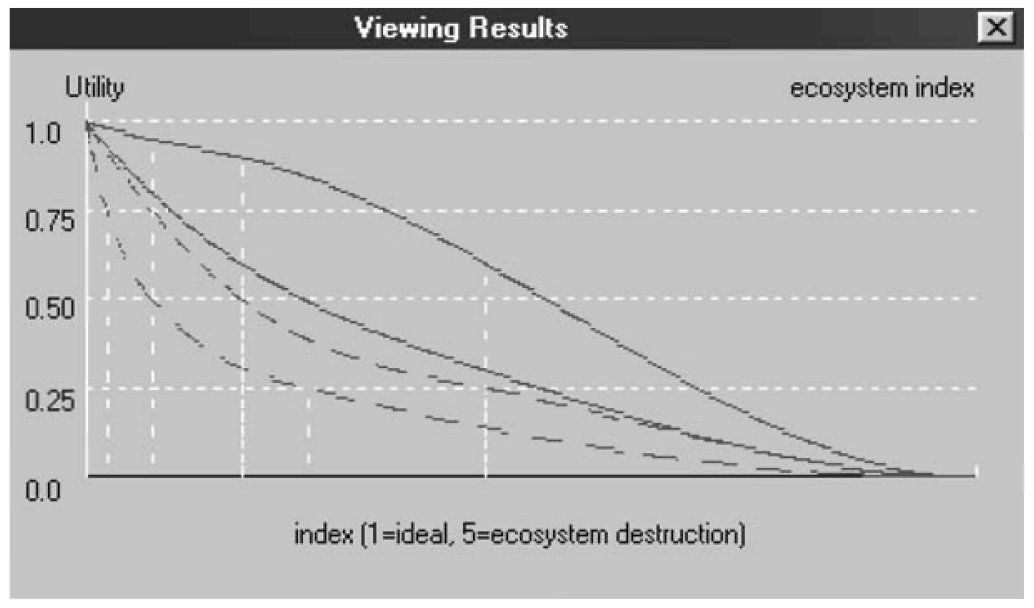

Figure 13. Classes of utility functions for Ecosystem Index.

weights could have been assigned taking into account their respective number of deputies or councillors. Their preferences were quantified separately, first they assigned component utility functions for the different attributes, which represent their preferences over their possible values, and then they elicited the objective weights in the hierarchy, which represent their relative importance. This leads to different classes of utility functions and weight intervals for each attribute under consideration, see, e.g., Figure 13 in which the classes of utility functions for the attribute Ecosystem Index are shown, or Figure 14, which shows the imprecise weights of the attributes over the decision for the two parties. The solid lines

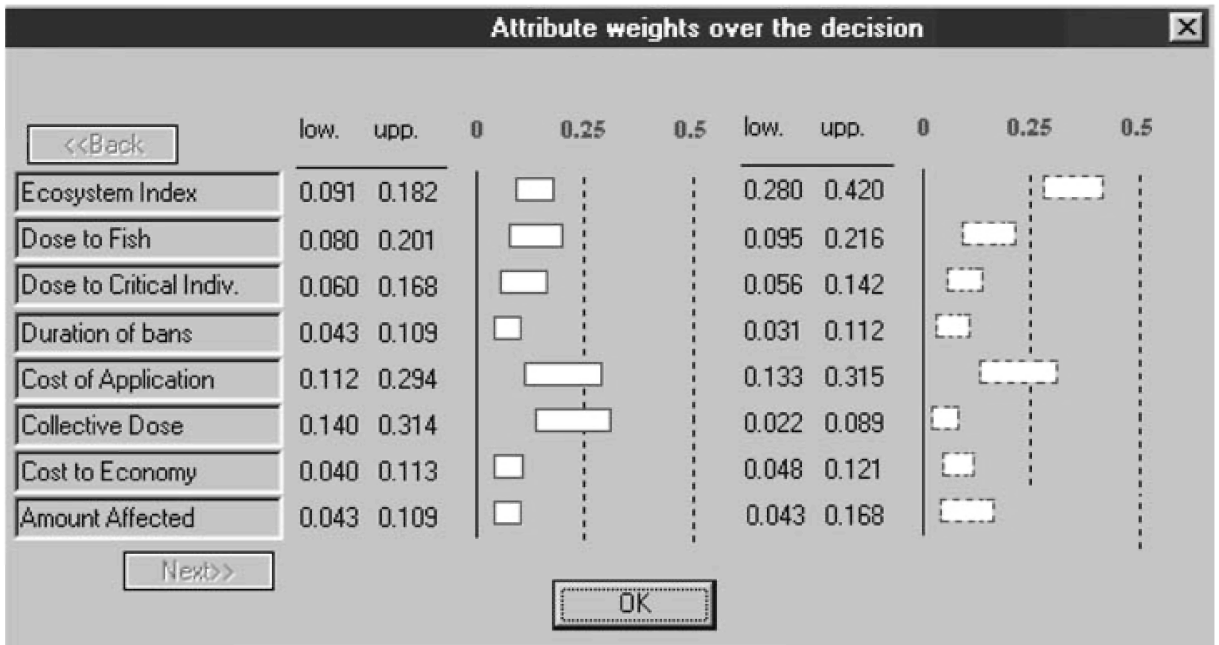

Figure 14. Weight intervals in the different attributes for the two parties. 
correspond to the first politic party, denoted from now on by the "solid" party and the dashed lines correspond to the "dashed" party.

Note that even if the most and least preferred attribute values are the same for both parties, as the utility functions represent the DMs' attitude towards risk, its appearance can be different, i.e., in an increasing utility function, if the DMs are averse to risk the utility function be will concave, while, in the case of risk prone DMs the utility function is convex.

Looking at Figure 14, we realize that the most important attributes for the "dashed" party are Ecosystem Index and Cost of Application, while they are Collective Dose and Cost of Application for the "solid" party. There are attributes where the intersection between the respective weight intervals is empty, like Ecosystem Index and Collective Dose, and attributes where the DMs' preferences almost completely match, like Cost of Application.

Once the group of DMs or parties have quantified their respective preferences separately, the simulation techniques are performed leading to the multiple boxplot and statistics shown in Figures 15 and 16, respectively.

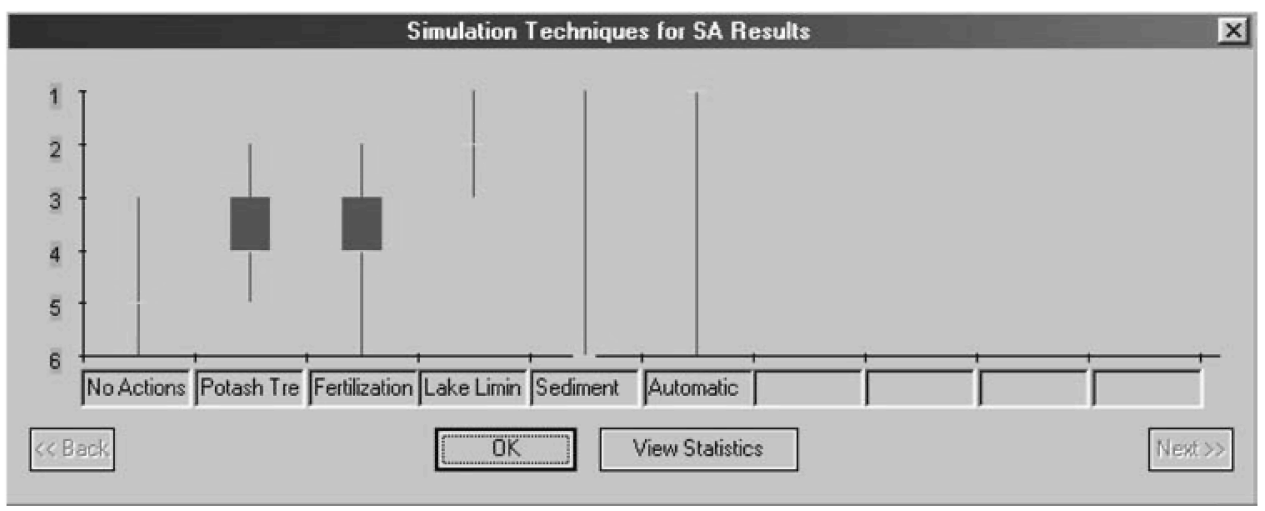

Figure 15. Initial Multiple boxplot.

\begin{tabular}{|c|c|c|c|c|c|c|c|c|}
\hline \multicolumn{9}{|c|}{ Statistics } \\
\hline & Mode & Min. & 25th percentile & 50 th percentile & 75th percentile & $\operatorname{Max}$ & Mean & Std. Deviation \\
\hline No Actions & 5 & 3 & 5.000 & 5.000 & 5.000 & 6 & 4.932 & 0.657 \\
\hline 曲Potash Treatment & 4 & 2 & 3.000 & 4.000 & 4.000 & 5 & 3.603 & 0.695 \\
\hline 曲 Fertilization & 3 & 2 & 3.000 & 4.000 & 4.000 & 6 & 3.772 & 0.847 \\
\hline 囲果 Lake Liming & 2 & 1 & 2000 & 2000 & 2.000 & 3 & 1.952 & 0.284 \\
\hline 曲 Sediment Removal & 6 & 1 & 6.000 & 6.000 & 6.000 & 6 & 5.530 & 1.062 \\
\hline Antomatic Food B... & 1 & 1 & 1.000 & 1.000 & 1.000 & 6 & 1.210 & 0.872 \\
\hline & & & & $\overline{O K}$ & & & & \\
\hline
\end{tabular}

Figure 16. Statistics associated to the initial multiple boxplot. 
Table 2. Divergence indexes for the first simulation.

\begin{tabular}{lllllllll}
\hline $\begin{array}{l}\text { Divergence } \\
\text { indexes }\end{array}$ & $X_{1}$ & $X_{2}$ & $X_{3}$ & $X_{4}$ & $X_{5}$ & $X_{6}$ & $X_{7}$ & $X_{8}$ \\
\hline $\mathrm{ADI}_{i}$ & 0.332 & 0.067 & 0.054 & 0.191 & 0.012 & 0.026 & 0.089 & 0.014 \\
\hline
\end{tabular}

In this multiple boxplot, three restoration strategies are best ranked for at least one combination of component utilities, weights and strategy consequences, Lake Liming, Sediment Removal and Automatic Food Bans, with mean classifications of 1.952, 5.530 and 1.210, respectively. It is clear that we must confine our attention to these three strategies. However, although mean rankings suggest the recommendation of Automatic Food Bans, it is worst ranked for at least one combination of the imprecise parameters, while the worst classification for Lake Liming is third. As the results are not meaningful enough to definitively recommend a consensus strategy, the negotiation process must be performed.

The attribute divergence indexes, $\mathrm{ADI}_{i}$ assessed in the simulation algorithm, are shown in Table 2. We can see that the attributes with the highest divergence indexes are Ecosystem Index and Collective Dose. Hence, the negotiation process must begin with these attributes. Now we pay attention to the imprecise component utilities and weights for these attributes. In Ecosystem Index the respective classes of utility functions for the two groups of DMs do not intersect at any point. The intersection between the respective weight intervals is also empty in both attributes. Therefore, the negotiation process focuses on them.

A meeting with representative of both parties was arranged, in which they actively discussed and changed their preferences. The discussion focused on the attributes Ecosystem Index and Collective Dose. The aim of this meeting was to achieve a nonempty intersection between the weight intervals in both attributes, see Figure 17, and a non-empty intersection in the whole range between the classes of utility functions in Ecosystem Index, see Figure 18.

The simulation process was rerun and the resulting boxplot is shown in Figure 19. Now, the information displayed is more meaningful, only two restoration strategies are best ranked, Lake Liming and Automatic Food Bans, with mean classifications of 1.944 and 1.159, respectively. Mean rankings again suggest the recommendation of Automatic Food Bans, but it is still worst ranked for at least one combination of the imprecise parameters. As a consequence, a decision was made to enter into further negotiations to definitively confirm this recommendation.

The attribute divergence indexes, $\mathrm{ADI}_{i}$, from the above simulation suggested revising the weight intervals corresponding to Cost of Application and Dose to Fish. The intersection between their respective weight intervals was not empty, but was too large. Hence the negotiation process involved reducing the intersection interval. There were no revisions of utilities, because the intersection between the respective classes of utility functions was not empty at any point for any attribute and the imprecision concerning the intersection classes was not high enough.

The simulation process was carried out again and the resulting boxplot and statistics are shown in Figures 20 and 21. Lake Liming and Automatic Food Bans are again best ranked, but the worst classification for Automatic Food Bans is now second. We decided to 


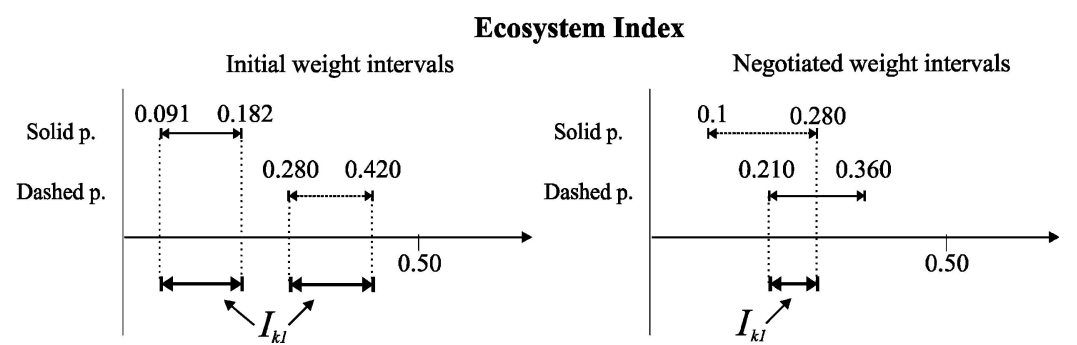

Collective Dose

Initial weight intervals

Negotiated weight intervals
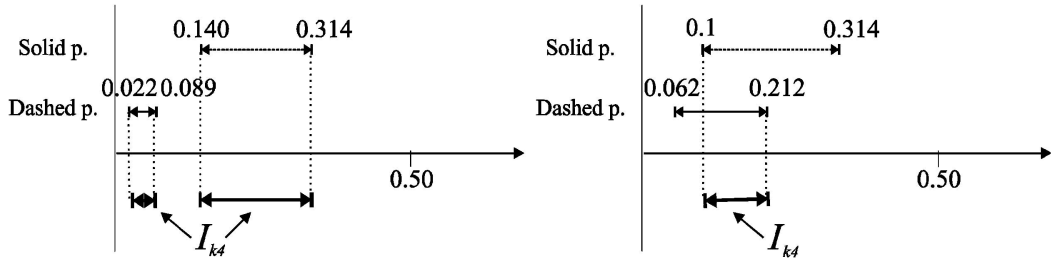

Figure 17. Negotiation process results in weights.

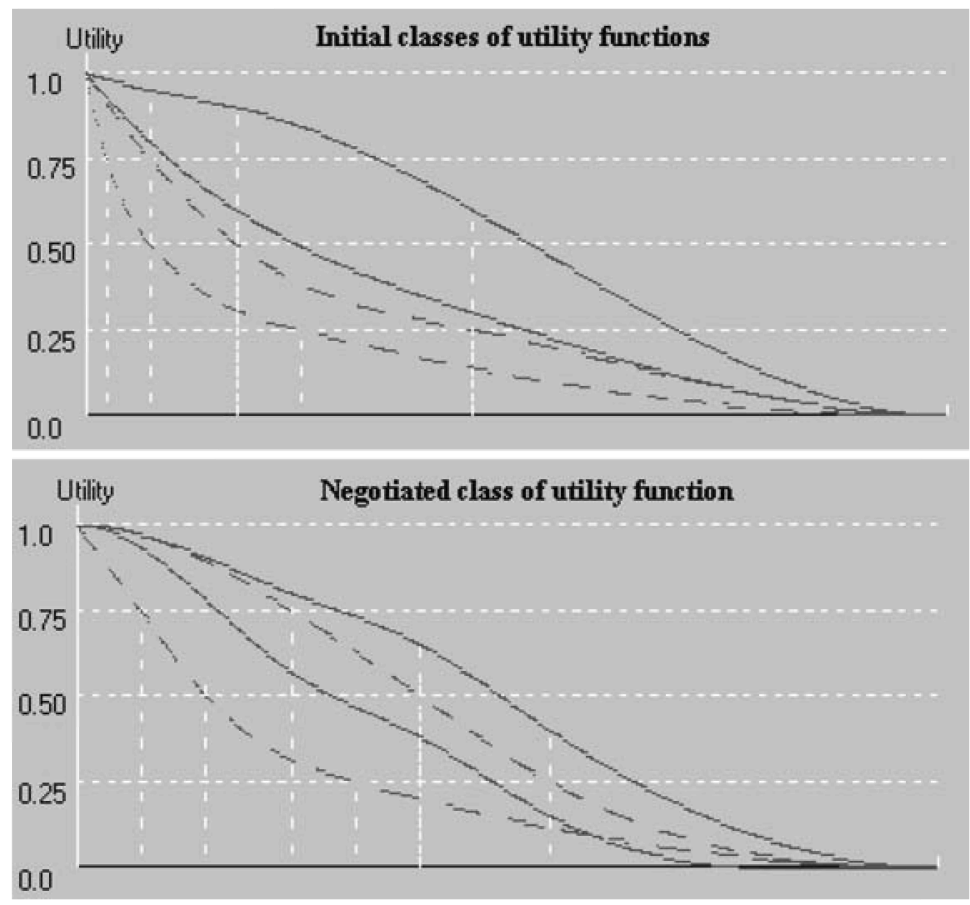

Figure 18. Negotiation process results for utilities. 


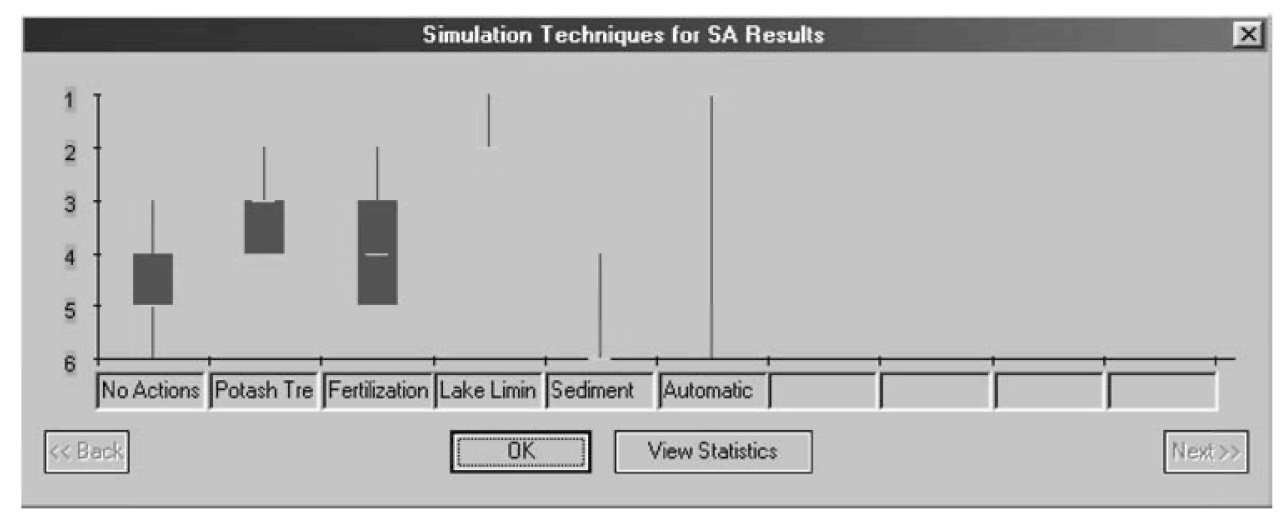

Figure 19. Multiple boxplot after the first negotiation.

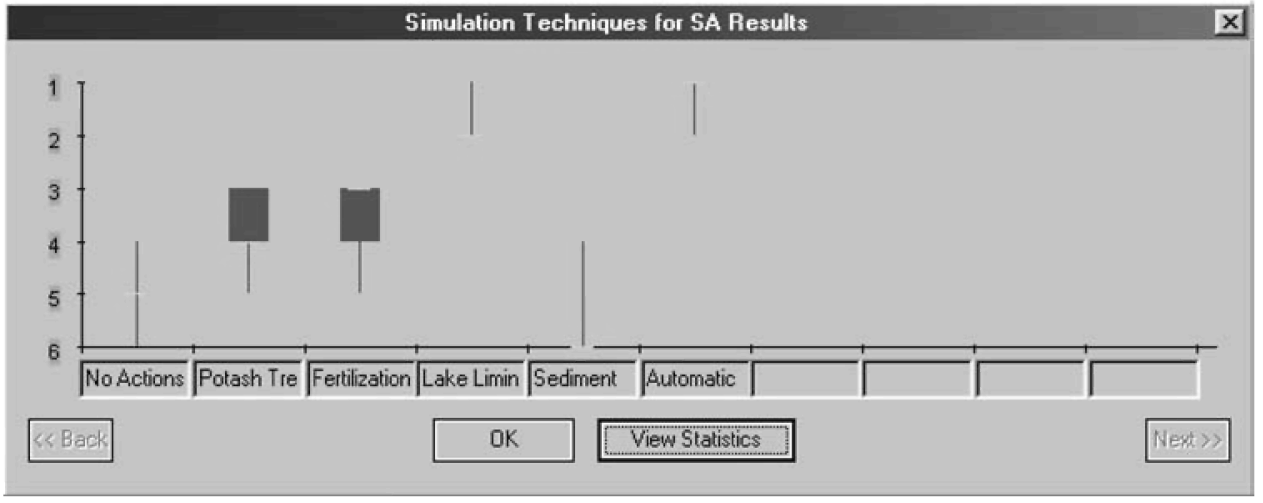

Figure 20. Final Multiple boxplot.

Statistics

\begin{tabular}{|c|c|c|c|c|c|c|c|c|}
\hline & Mode & Min. & 25th percentile & 50th percentile & 75th percentile & Max & Mean & Std. Deviation \\
\hline No Actions & 5 & 4 & 5.000 & 5.000 & 5.000 & 6 & 4.893 & 0.472 \\
\hline Potash Treatment & 4 & 3 & 3000 & 4.000 & 4.000 & 5 & 3.584 & 0.512 \\
\hline \#曲Fertilization & 3 & 3 & 3.000 & 3.000 & 4.000 & 5 & 3.596 & 0.763 \\
\hline 曲国Lake Liming & 2 & 1 & 2000 & 2.000 & 2.000 & 2 & 2.000 & 0.010 \\
\hline Wediment Removal & 6 & 4 & 6.000 & 6.000 & 6.000 & 6 & 5.927 & 0.295 \\
\hline 曲西 Automatic Food B... & 1 & 1 & 1.000 & 1.000 & 1.000 & 2 & 1.000 & 0.010 \\
\hline
\end{tabular}

Figure 21. Statistics associated to the final multiple boxplot. 
definitively recommend it on the basis of the mean values corresponding to both strategies, 1.0 and 2.0 , respectively.

\section{Conclussions}

We have introduced the different procedures provided by a DSS based on an additive multi-attribute utility model, the GMAA system, for quantifying DM preferences. The system accounts for incomplete information on the component utility and weight elicitation, through ranges or intervals of responses. This is less demanding for a single DM and makes the system suitable for group decision support, because individual conflicting views or judgements in a group of DMs can be captured through imprecise responses.

The system is used in a group decision-making situation to quantify the DMs' or group of DMs' preferences separately. Hence, several classes of utility functions and imprecise weights are available for each attribute under consideration. An approach based on Monte Carlo simulation techniques has been proposed as a starting point for a negotiation process. Throughout the simulations, strategy consequences and their associated utility and attribute weights are generated at random taking into account the imprecise strategy consequences, the imprecise component utilities and weights intervals, respectively. A negotiation process can be carried out on the basis of the multiple boxplot displayed when the simulation is run and from the attributes, either the imprecise component utility functions or weights intervals or both on which the DMs' or groups of DMs' preferences agree less.

The application to the restoration of aquatic ecosystems contaminated by radionuclides shows the usefulness and flexibility of the iterative process. The tighter imprecise component utilities and weights are, based on divergence indexes, the more meaningful the information displayed in the multiple boxplot will be. Finally, a consensus strategy is reached.

\section{Acknowledgement}

This paper was supported by the Ministry of Education and Science TSI 2004-06801C04-04. (1) http://www.dia.fi.upm.es/ ajimenez/GMAA.

\section{References}

Bratley, P., B. L. Fox, and L. E. Schrage. (1983). A Guide to Simulation. New York: Springer-Verlag. Bui, T. X. (1987). “Co-oP: A Group Decision Support System for Cooperative Multiple Criteria Group Decision Making", in Lecture Notes in Computer Science 290. Berlin: Springer-Verlag.

Ethamo, H., M. Verkama, and R. P. Hämäläinen. (1994). Negotiating Efficient Agreements over Continuous Issues. Research Report A51. Systems Analysis Laboratory, Helsinki University of Technology.

Farquhar, P. H. (1984). "Utility Assessment Methods," Management Science 30, 1283-1300.

Fishburn, P. C. (1964). Decision and Value Theory. New York: Wiley. 
Fishburn, P. C. (1970). Utility Theory for Decision Making. New York: Wiley.

French, S. (1986). Decision Theory: An Introduction to the Mathematics of Rationality. Chichester: Ellis Horwood. French, S., K. N. Papamichail, D. C. Ranyard, and J. Q. Smith. (1997). Design of a Decision Support System for Use in the Event of a Nuclear Accident. RODOS Report (WG5)-TN(97)-04.

Gallego, E., A. Jiménez, A. Mateos, T. Sazykina, S. Ríos-Insua, and M. Windergärd. (2001). "Application of Multiattribute Analysis Methodologies to the Evaluation of the Effectiveness of Remedial Strategies with the MOIRA System", in L. Monte, J. E. Brittain, L. Häkanson, E. Gallego, M. Zheleznyak, Oleg Voitsekhovitch, I. Krishev, and K. M. Petrov (eds.), Implementing Computerised Methodologies to Evaluate the Effectiveness of Countermeasures for Restoring Radionuclide Contaminated Fresh Water Ecosystems. Rome: ENEA.

Hämäläinen, R. P. (1996). “On-Line Group Decision Support by Preference Programming in Traffic Planning," Group Decision and Negotiation 5, 485-500.

Holloway, C. A. (1979). Decision Making Under Uncertainty: Models and Choices. New Jersey: Prentice-Hall.

Hull, J. C., P. G. Moore, and H. Thomas. (1973). 'Utility and Its Measurement', Journal of the Royal Statistical Society 136, 226-247.

Hwang, C.-L. and M.-J. Lin. (1987). Group Decision Making Under Multiple Criteria. Berlin: Springer-Verlag.

Iz, P. H. and L. R. Gardiner. (1993). "Analysis of Multiple Criteria Decision Support Systems for Cooperative Groups," Group Decision and Negotiation 2, 61-79.

Jarke, M., M. T. Jelassi, and M. F. Shakun. (1987). "MEDIATOR: Towards a Negotiation Support System," European Journal of Operational Research 31, 314-334.

Jiménez, A. (2002). Un Sistema de Ayuda a la Decisión Multiatributo con Asignaciones Imprecisas, Ph D. Dissertation. Technical University of Madrid.

Jiménez, A., S. Ríos-Insua, and A. Mateos. (2003). "A Decision Support System for Multiattribute Utility Evaluation Based on Imprecise Assignments," Decision Support Systems 36(1), 65-79.

Keeney, R. L. and H. Raiffa. (1976). Decision with Multiple Objectives: Preferences and Value-Tradeoffs. New York: Wiley.

Mateos, A., A. Jiménez, and S. Ríos Insua. (2003). "A Multiattribute Solving Dominance and Potential Optimality in Imprecise Multi-Attribute Additive Problems', Reliability Engineering and System Safety 79, 253-262.

Mosteller, F. and P. Nogee. (1951). “An Experimental Measurement of Utility," Joumal of Political Economy 59, $371-404$.

Mumpower, J. L. (1991). "The Judgement Policies of Negotiators and the Structure of Negotiation Problems," Management Science 37, 1304-1324.

Ríos-Insua, D. (1994). "Ambiguity, Imprecision and Sensitivity in Decision Theory," in J. P. Vilaplana and M. L. Puri (eds.), Recent Advances in Statistics and Probability. VSP.

Schlaifer, R. (1969). Analysis of Decisions Under Uncertainty. New York: McGraw-Hill.

Verkama, M., R. P. Hämäläinen, and H. Ethamo. (1992). "Multi-Agent Interaction Processes: From Oligopoly Theory to Decentralized Artificial Intelligence," Group Decision and Negotiation 2, 137-159.

Verkama, M., R. P. Hämäläinen, and H. Ethamo. (1994). "Modeling and Computational Analysis of Reactive Behaviour in Organizations," in K. M. Carley and M. J. Prietula (eds.), Computational Organizational Theory. New Jersey: Hillsdale.

von Nitzsch, R. and M. Weber. (1988). "Utility Function Assessment on a Micro-Computer: An Interactive Procedure," Annals of Operations Research 16, 149-160. 